\title{
Georgij Yu. Somov \\ The types of codes and their combinations: Visual perception and visual art
}

\author{
Postprint ${ }^{*}$ v.1.0 \\ ${ }^{*}$ Somov, G. (2014). The types of codes and their combinations: Visual perception and \\ visual art. Semiotica, 2014(202), pp. 481-509. Retrieved 7 Oct. 2017, from doi:10.1515/ \\ sem-2014-0041
}

\begin{abstract}
Visual perception and visual connection are implemented thanks to many codes. Formation of code typology is one of the problem of semiotics. This typology may be substantiated ontologically - through universals of heterogene-ity of visual art works. Groups of elements and relations of heterogeneity that are connected with structures correspond to various codes. In accordance with this fundamental types of codes, their subtypes and combinations are allocated. The suggested chart of code typology describes the differences and peculiarities of language-type systems and texts of visual information and visual art.
\end{abstract}

Keywords: signs, semiotic systems, structures, pre-language, nonverbal systems, visual information

The traditional way to describe the semiotic concept of visual art, i.e., "codes languages - texts - sign formations of artworks," requires an interpretation of codes and related concepts. First and foremost, it's necessary to explain the differentiation between codes and the notion of "languages," which is close to them. The need for this distinction is based on a great number of code definitions and codes and languages that have been broadly discussed in relation to visual art (Gombrich 2000 [1960]; Zemsz 1967: 59; Eco 1968; Schapiro 1969; Damisch 1978; Nöth 1998). Otherwise stated, the concepts of codes and languages themselves need to be developed in non-verbal semiotic systems.

The term "language" is appropriate to interpret within the general concep-tion of semiotic systems of visual art on the basis of codes. This corresponds with the general tendency of dissemination of codes as the fundamental concepts of semiotics (“the landslide effect”; Eco 1984: 166). This also follows from the fact that the concept of code is a natural general semiotic and has the ontological status of universals. Codes are universals that are present in the entire life of signs - the DNA, sensor information, technical systems, etc. In addition, codes, 
i.e., the most abstract and universal objects of semiotic systems, are interrelated in some semiotic theories with concepts of structures, dual structures and features, and types of signs. Owing to their abstract character, codes are objects of many semiotic-related sciences (or branches of semiotics): information theories, genetics, neurophysiology, neurolinguistics, etc. That is why the notion of "codes" in its interrelation with structures and other highly abstract concepts can serve as a foundation for the ontological description of the semiotic field being investigated. Other semiotic concepts - languages, texts, contexts, metaphors - can be interpreted on the basis of codes.

As explained below, codes need to be regarded largely in their interrelation with the concepts of "heterogeneity," "structure," "elements," "relations" and other relative concepts of the system-structural approach. This corresponds to the definition of codes as structures in semiotics (Prieto 1975), the idea of structures as the center of semiotic systematicity by Hjelmslev. This area of semiotics as any scientific area creates the need for an ontological substantiation of these abstract concepts and the reality of universals. Previously, the author has tried to show the reality of codes and other related abstract objects (structure, relations, elements, etc.; Somov 2012).

The notion of "codes" is defined as "sign systems," "structures," "mechanisms." Based on differentiation between these notions, codes should be regarded as mechanisms of transformations, while systems, structures, and other universals mentioned above - as manifestations of these mechanisms in heterogeneities. At the same time, codes are inseparable from various universals of heterogeneities. This allows for consideration of manifestations of various types of codes in images. Groups of elements and relations of heterogeneity that are connected with structures correspond to various codes. Thus, groups of elements and relations of an image can be specified based on the assumption that they correspond to a certain code. If these groups of elements and relations are typical for a number of pieces of art, they correspond to paradigmatic codes. If they are typical for a single piece of art, they correspond to codes of structures of expression of this work - a text. There are exist various classifications of codes of semiotic systems (Buyssens 1967 [1943]; Mounin 1970; Eco 1968; Fabbri et al. 1976: 167, 203, 222; Guiraud 1975). The classification suggested by Eco in "The Absent Structure" (Eco 1968) is the most detailed one. Semiotics is full of new ideas of code classification based on the development of post-structuralist conceptions and related sciences. However, a single typological outline connecting the most important classifications of codes is needed to provide a holistic description of semiotic systematicities in visual art. That is why only classifications and conceptions related to the suggested outline are mentioned below out of all existing ones. 
The key peculiarities of the aforementioned content of codes should be specified in the system of the existing semiotic definitions. Based on the statements formulated above, a typology of codes needs to be proposed in which works of visual art are formed, implemented and evolve. Respectively, below are the differentiations between types of codes. These types of codes are fundamental and include subtypes that are relatively independent from each other. Basic interrelations between types of codes are presented in a scheme (Figure 1).

Codes have double articulation. They (Figure 1, group 1) are implemented in sign systems at various levels. According to Hjelmslev, there exists a division into figures of plane of expression and figures of plane of content (Nöth 1998: 215-216, see subtypes of double articulation codes in Figure 1). Hence, three levels are formed: figures, signs, and semes (Prieto 1964). These distinctions reflect the idea of the three levels of construction in the theory of artificial languages by Garwin.

Based on the terms of the theory by Prieto, it would be reasonable to distinguish between figures or signals (a), signs (b), and semes or semantic components (c). In visual information and visual art, codes of double articulation (as in other processes of semiosis) form relations between heterogeneities: $b \leftrightarrow a$ and $b \leftrightarrow c$. Due to double articulation, the heterogeneity of elements and relations perceived visually is connected with the heterogeneity of signs and the heterogeneity of semantic level. Visual perception can catch meaningful visual features (geometric bodies, colors, textures, etc.); meaningful sign features (contours of people and objects, color features of difference between these designates); meaningful semantic features (most important objects, their structures in certain life situations, structures of their importance in paradigmatics, etc.) at the same time. Based on double articulation, relations between the given levels are realized in visual information - in an image. Thus, a danger (semantic component) is expressed in dynamics (sign), as well as slopes, sharp angles, heavy moving masses (representamens and their typical features). A sign - a silhouette - contains an object - a figure of a running person (seme), as meaningful features of a silhouette correspond to certain features of a human. Double articulation is also implemented in image organization. This corresponds to the three levels of visual signs (Eco 1968). Organizing structures of important slopes, heavy moving masses, figures of running people (structures of signs) are formed. Configurations, contours, axes, centers, color areas of these elements (structures of their representamens) are organized. Structures of directions of movements, energies, relations between people (structures of semantic elements) are formed.

Codes are mechanisms of translation and transformation of structures, their interaction and combination. Ideas of translation of structures are represented 


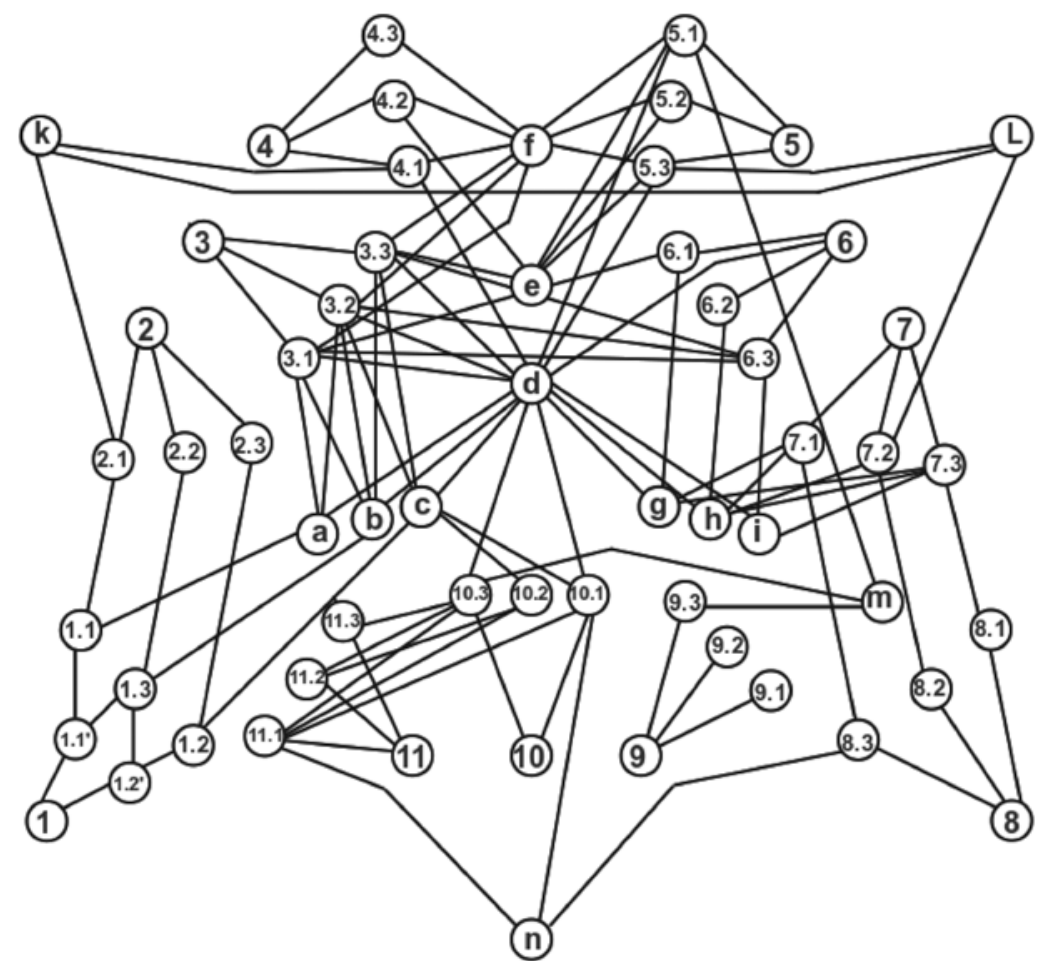

Fig. 1: Main interrelations between basic types of codes. Types and subtypes of codes: 1 - double articulation codes, $1.1^{\prime}$ - first articulation, expression-plane, $1.2^{\prime}$ - second articulation, content-plane, 1.1 - codes without second articulation, 1.2 - codes without first articulation, 1.3 - codes with first and second articulations; 2 - codes of transformations of structures, 2.1 - codes of translation of structures, 2.2 - codes of structural interactions, 2.3 - codes of structural combinations; 3 - codes of sign relations, 3.1 - intentional codes, 3.2 - identification codes, 3.3 - organizational codes; 4 - codes of the sign triad, 4.1 - iconic codes, 4.2 - indexical codes, 4.3 - symbolic codes; 5 - evolutional codes, 5.1 - natural (acquired) codes, 5.2 - situation codes, 5.3 - cultural codes; 6 - paradigmatic-text codes, 6.1 - paradigmatic codes, 6.2 - codes of structural expression in text, 6.3 - codes of discourses; 7 - codes of language systems, 7.1 - pre-language codes; 7.2 - language codes, 7.3 - codes of language expression; 8 - codes of communication channels, 8.1 - nonverbal codes, 8.2 - verbal and linguistic codes, 8.3 - visual codes; 9 - regulatory codes, 9.1 - codes of information selection, 9.2 - codes of response, 9.3 - codes of actions; 10 - codes of integration levels, 10.1 - codes of universals, 10.2 - codes of bundles and realizations, 10.3 - codes of patterns; 11 - codes of different realities, 11.1 - codes of life reality, 11.2 - codes of images, 11.3 - codes of virtual realities. Typical combinations of codes: a - figures or signals; b - signs; $c$ - semes or semantic components; $d$ - icons; $e$ - indices; $f$ - symbols; $g$, $h$, and $\mathrm{i}$ - semiotic systems of different levels; $\mathrm{k}$ - identification codes of icons on the base of translation of structures; l - cultural paradigmatic language codes; $\mathrm{m}$ - paradigmatic codes of patterns' actions; $\mathrm{n}$ - codes of universals of visual perception of life reality. 
in semiotic theories (Barthes 1965; Stepanov 1971; Lefebvre 1973 [1967]). The idea of interaction of structures is reflected in the mathematical structuralism of the Bourbaki Group (Aczel 2006) and philosophical problems of structures in psychology (Mouloud 1965, 1969; Piaget 1968). Thanks to a structural basis all codes can be presented digitally. This corresponds to the idea that analog codes in their structure are digital (Hoffmeyer and Emmeche 1991). Form-building models in architecture (Barbyshev and Somov 1972) are also close to these ideas.

Various codes are differentiated in transformation of structures. Hence, it would be reasonable to distinguish the following codes among the codes of transformations of structures (Figure 1, type 2): codes of translation of structures (2.1), codes of structural interaction (2.2), codes of structural combinations (2.3). Codes of structural translation (2.1) are manifested in various techniques of organization of pieces of visual art. For instance, they include the techniques of rotationreflection symmetry of meaningful parts: top and bottom, left and right parts of an image (Somov 2005, 2007b). Translation of symmetry is manifested as identity of the aforementioned parts. A sort of a mirror is formed. A single system of dimension ratio (proportions), including with the use of geometrical similarity of rectangles, is a manifestation of structural translation as well. Similarly, the number of elements is repeated in a different group of elements. In particular, these techniques determine the techniques based on structures of two $(2+1)$, three $(3+1)$, four (4+2), and five (5+2) elements (Somov 2008). Codes of interaction (combination of structures) (2.2) are mechanisms connecting various structures. Structural interaction is performed in bundles (realizations). The idea of bundles of differential features was originally developed in the theory of phoneme (Trubetzkoy 1969 [1939]) and in theoretical generalization in semiotics (Jakobson 1976). Such bundles or realizations became the core element of modelling in the theory and methods of image recognition (Rosenfeld 1969; Bongard 1967; Fayermark 1974). Their models relating to visual perception were developed in particular detail (Bongard 1967). A number of works regarded the relation of these models to semiotics and psychology (Shekhter 1967). In models of image recognition, elementary features are integrated into some combinations (bundles or realizations), according to which identification is carried out (identification codes [3.2] are realized). Identification of silhouettes can serve an example of structural combinations that form bundles or realizations. This identification is performed according to their structural features (topological structures: closed-open, salient-concave, configurations that have either broken contours or smooth curves; symmetries: reflectional symmetry-no axes of symmetry; numerical structures: numbers of elements in various groups of elements and relations, etc.). The combination of these differential structural features allows for matching them with a certain 
class of objects. These interactions serve the basis of mechanisms responsible for coding and decoding of the basic signified. The simplest natural codes are described similarly to the given models (certain locations of objects in space on the basis of dimension ratio, identification of an object on the basis of individual features; Somjen 1972). Unification of various structures and structural features enables both identification (relations between the signifier and the signified) and organization. The use of certain techniques illustrates this point in the formation of images. Thus, interaction between two different systems of proportions creates a common system, for example, one combining two types of geometrically similar rectangles. This simple technique turns out to be very important for the formation of visual information. Systems of geometrical similarities that organize a vast area of an image are revealed in the course of semiotic analysis. They connect the main signs in a painting according to their importance and emphasize the core element of an image. In particular, large, multi-figured paintings of symbolic nature are organized in this way (Somov 2005). Thus, code 2.2 seem to direct interactions and combinations of structures, with the participation of various structures (dimensional, topological, symmetries, numerical and other). Codes of structural combinations (2.3) direct transformations of sign formations into other sign formations, from one sign to a fundamentally new sign. Combinations are connections that unite diametrically different types of structural organizations. In particular, groups of elements that are scattered unexpectedly or randomly are opposite to order. Scientists usually refer to relations between picturesqueness (disorder) and order. This resonates with some mathematical descriptions of these formations by quantitative methods of information. Some fragments, parts, systematicities can be characterized as chaotic, while others can be described as ordered. The way one turns into another is determined by a special structure - a special code. The techniques of organization of visual information, images, pieces of visual art in which these codes are manifested are multifarious. For example, large areas of an image are sometimes organized as integral color configurations with their own structures of topology, dimensions, numbers, etc. Each of these areas contains complicated chaotic groups of elements. Generally, such technique can be called a "mosaic." This method specifies a certain principle of image perception - the principle of semiosis. An image can be perceived either as an entire sign system of major iconic signs, or separately - as a chaotic filling of each area containing a group of elements in it. A structural code operates in the production of such information: it serves to make some structures, features, elements autonomous from the other and to facilitate the creation of various kinds of perception - semiosis of sign groups.

The relations between structures and types of signs have been repeatedly discussed in semiotics (Stepanov 1971; Sonesson 1997). In our opinion, the afore- 
mentioned transformations of structures are interrelated with the properties and types of sign relations. Codes of structural translation (2.1) are realized in icons; codes of interaction (combinations) (2.2) are realized in icons and indices; codes of transformations (2.3) are realized in icons, indices, and symbols.

Codes of structural transformations (2) have been illustrated above by elements and relations of visual level. However, translations, interactions, and combinations of structures include various relations between the signifier and the signified and various levels of construction. In particular, the duality of codes is reflected in the duality of structures. Otherwise stated, dual structures of heterogeneity of the signifier specify dual structures of heterogeneity of the signified. That is why structures of sign systems are present at different levels of the psyche: at levels of senses, images - perceptions, concepts, feelings, etc. Such are the essential oppositions of good and evil, fairness and unfairness, truth and lie. This duality and its universal nature correlate with the conceptions of semantic structures (Greimas and Courtes 1979), dual structures in anthropology (Ivanov 2008). Thus, codes of transformations of structures embrace all levels of construction and apparently all spheres of sign systems.

The distinction between intentional identification organizational codes is implemented in various aspects (dimensions) of sign systems - in sign systems of pragmatics, semantics, and syntactics. The difference between these codes needs to be clarified based on a model of a sign, according to Peirce. Codes connect various aspects of a sign. According to Peirce's model of a sign, these codes can be represented as relations between the model's elements: representamens and interpretants, objects and other representamens (Figure 1). In this group of codes, sign systems are implemented in relations: 1) interpretants-representamens (pragmatics); 2) objects-representamens (semantics); 3) representamensrepresentamens (syntactics). These relations develop. This is similar to how codes are viewed in semiotics, where they are usually regarded within the "signifier-signified" relations. Hence, numerous interpretations of codes connect them with the "signs-semes" relations by conceptions of Buyssens and Prieto. This is a modification of the "signifier-signified." The classification of codes proposed by Eco resulted in the need to distinguish between semantic and syntactic codes, semantic and musical (aesthetic) codes, regarding codes as rules of relations in sign systems (Eco 1968). Musical order is a code. Obviously, this order is of aesthetic nature, but in other sign systems it can be related to resolving certain thinking problems. In semiotic categories, these codes correspond to relations between elements of semiotic systems (RR), i.e., to syntactics. In turn, if codes are differentiated in accordance with semantic and syntactic aspects, it would be logical to introduce the pragmatic aspect as well and consider types of codes in the three semiotic aspects. 
Thus, codes of sign relations (Figure 1, type 3) are divided into subtypes according to the types of relations: R-O-I, R-O, and R-R. Intentional codes (3.1) (R-O-I) connect intepretants and representamens. To classify them, one needs to differentiate between interpretants. Elementary interpretants include the following: needs (instinctive desires, according to Kristeva, general motives - individual intentions). General intentions are typical for animals and people: the need for preservation and the related urge towards safety, search for food, partners, moving in space, co-participation. Obviously, there are certain peculiarities and substantial differences between human and animal fundamental needs (intentions). Some general intentions and intentional codes, though, are of general biological nature. Identificational codes (3.2) - codes that connect representamens and objects are described in detail in various semiotic research (Eco 1968). They can be interpreted as the signified-signifier relations where objects are the signified. These codes ensure identification of these or those objects. There are also exist organizational codes (3.3) that connect representamens $(R-R)$, objects, and interpretants $(\mathrm{O}-\mathrm{O}, \mathrm{I}-\mathrm{I})$; and signs with other signs $(\mathrm{Sn}-\mathrm{Sn})$. Otherwise stated, they connect parts and the whole in a certain structure and organize information processes. In visual art there are structures, features, differences, and identities that organize elements and relations, i.e., universalia that form the basis of codes. They include, in particular, obvious universalia, namely: symmetries, rhythms, numbers, centers, axes and contours, color formations, geometric configurations, bodies, etc. These organizing universalia have been traditionally related to aesthetic perception. They play an important role among aesthetic phenomena and are naturally generalized in the term "aesthetic codes" (Eco 1968). This term needs to be specified, though. To identify the manifestations of codes of mental processes with aesthetic processes means to follow traditions but thus the problem becomes simplified. Artistic and aesthetic phenomena can be related to the system of values (pragmatics; Lukács 1969), with particular structures of literary texts (Lotman 1977), with various connotations of aesthetic sign (Morris 1971 [1939]), with manifestations of global symbols (Cassirer 1965), and with specific types of meanings by Whitehead (1927) and aesthetic information by Bense (1982 [1965]). Otherwise stated, in a number of conceptions the manifestations of aesthetic perception of artworks are regarded broader than the manifestations of codes of mental processes. Hence, the accepted term "organizing codes" would sound more accurate to define the aforementioned codes. These codes function in the organization of groups of visual elements, features, and relations. For example, axes promote the relations between bodies, contours - the relations of boundaries, rhythms of color spots - the formation of a single color range as well as rhythmical structures of an image. These unifying structures, features, and identities help form codes of relations between groups of elements, carry out 
mental processes of identifying structures in complicated heterogeneities as well as forecasts like "expected-unexpected" in information processes.

According to the classification of signs elaborated by Peirce, i.e., "primarysecondary-tertiary nature," the following levels are singled out: 1) property, icon, rheme; 2) individual, index, judgement; 3) general, symbol, conclusion (Nöth 1998). To make the distinction between codes easier, the author describes their manifestations in the "icon-index-symbol" relations. Thus, codes of the sign triad (Figure 1, group 4) include iconic codes (4.1), indexical codes (4.2) and symbolic codes (4.3). This trichotomy reflects the way iconic codes are specified and regarded (Eco 1968). Iconic codes (4.1) are mechanisms that connect qualities. Any connections of homogeneous qualities are the basis of iconic codes. Color configurations that remind one of people form an iconic code in an image, in which people are identified on the basis of general features of signs and representamens (Eco 1968). Complicated iconic formations are produced on the basis of various codes. Special relations - special iconic codes - are established between these formations, which is clearly seen in the identification and depiction of people. Features of head, face, hands, mimicry, look, and other elementary features correspond to simple codes. Based on numerous features and functioning of numerous codes, the features that enable human identification are formed and function in an image. Iconic codes that unite simpler codes are formed. Evidently, the formation of these integrating codes is influenced by discourses and is not a mere mechanical unification of "simple codes" (Dijk 1982, 1987). Indexical codes (4.2) are inseparable from indices. These are mechanisms that connect the signifier (representamen) with what it designates. Indexical codes are formed due to natural connections. The significance of these relations in semiotic studies of culture was shown specially (Barthes 1968). A bright color spot means a flash, explosion, flame. An active line designates force direction and energy. Stable codes of such connections become essential in sign systems used by great masters and various schools of visual art, as these codes are specific for the production of sensor information. The designation of force or motion directions, space extension or narrowing, pressure or flight are typical codes of indices that generate pivotal meanings in visual art. Indexical codes enable one to identify a situation, to see the causeand-effect relationship between objects, their interaction. These codes reflect life situations in a way. A gesture of a person who raised his/her hand against somebody is an important index in life. It turns into a code. Hence, any hint of a raised hand becomes an essential sign in an image. Similar is the gesture of protection against an assaulter. These indexical codes are typical for images regardless of the culture in which these images were created. There also exist numerous indexical codes that are formed within the framework of certain traditions of visual art. Fighting soldiers who blend with the horses form an independent code in visual 
art (soldiers in Leonardo's The Battle of Anghiari', in paintings by Rubens, in paintings of battles and scenes of hunting by Delacroix). Later, in the nineteenth and twentieth century art a similar code is produced by an index pattern where a racing rider designates escape, pursuit, coming to succor or rushing to pass important news. Therefore, features of the rapid movement of horses carrying riders become significant codes in works by masters of visual art. Complicated sign formations of indices are formed in the combinations of features of indexical codes. In particular, human emotional states or actions that guide a person or a group of people can be revealed based on combinations of features of moving directions, poses, gestures, face expression. This integration is realized by codes that enable one to discern hidden inner states, thoughts, intentions, passions. The connections between these codes form structures of life situations. On the whole, they take part in the formation of judgments in terms of the sign theory elaborated by Peirce. "Visual judgments" are produced in visual art due to indexical codes (Arnheim 1974). Symbolic codes (4.3) realize indirect conventional connections that supplement icons and indices. These codes help interpret and connect symbols with each other. An image contains a set a symbols in simple sign systems that are similar to written language. Qualities, elements, relations of these symbols are the basis of symbolic codes in a given image or a number of similar images. Conventional sign systems used in icon painting and the related symbolic codes are the most obvious. According to semiotics they form system of symbols with syntax and lexical dimension (Schapiro 1969; Uspensky 1976). Such are the canons of color and color configurations in Byzantine icons. Each color area is connected to a verbal symbolic code. Thus, the dark cherry kerchief worn by Mother of God meant sacrifical love (Lazarev 1997), white and yellow-white elements symbolized the divine energy of light (Onasch 1962, 1988). Blue and its tints signified the spiritual and the Holy Spirit. The combination of symbolic colors with each other and with symbols of structural construction (triangles of the Holy Trinity, the space circle, the sacrificial chalice, configurations of the sacrificial flame, etc.) enabled the symbolization of the triunity of the world.

Symbolic codes (4.3) supplement icons and indices. Iconical and indexical codes connect the signifier and the signified directly. Symbolic codes connect them in a conventional-indirect way. This accounts for a well-known property of sign systems, symbols - to form temporal (intensive) structures and to be combined in an independent way. Therefore symbolic codes of visual information are closely connected with the development of written language and speech. They develop as

1 Peter Paul Rubens's copy of Struggle around the Standard from The Battle of Anghiari by Leonardo (1550-1603, black chalk and ink heightened with lead white, overpainted with watercolor, Louvre, Paris). 
codes of structural transformations and are involved in the modelling of changes. In life situation these processes are fulfilled in the structures of mental models in "deductions," in terms of the sign theory elaborated by Peirce (Nöth 1998).

The distinction between natural and acquired codes that was formulated in some works on semiotics enables the regarding of more subtle interrelations of instinctive desires, elements of mythological thinking, rules that are formed in culture, sensor processes and verbalized perceptions as well as a number of other essential semiotic systems of visual information and visual art. In particular, identification of physical properties of depicted objects is based on relations properties of real objects with features of depicted objects. Hot, warm, cold, wet, dry are felt due to innate codes. Many proper artistic phenomena of visual art and visual information are based on sign systems of designating similar properties and on sensor information coding. In semiotic research on visual information and art, codes cannot be confined by area of semiotic systems of culture, a fortiori by influence of the verbal sphere. The notion of "codes" needs to be expanded to the sphere of innate codes. The differences "cold-warm," "dangerous-harmless" are of innate nature. Codes of identification of these referents generally expand to sensor codes of many animals (Somjen 1972). At the same time, there are numerous non-innate codes. Hence, it would be reasonable to distinguish codes of evolution levels (Figure 1, type 5): natural and acquired codes (5.1), situation codes (5.2), and cultural codes (5.3). The differences between these evolution codes are revealed in general semiotic models: $5.1 \leftrightarrow 5.2$ and 5.3 as "egg-chicken" (Hoffmeyer and Emmeche 1991); $5.2 \leftrightarrow 5.3$ as important vital situations and all semiotic systems of interindividual communication. The differentiation "innate codes"-“codes of situations"-“cultural codes” helps understand the development of codes - their cycles in evolution of an individual and/or society. Once works of visual art and their formations are perceived, processes are fulfilled that integrate natural and cultural types of semiosis and mechanisms - codes. Dangerous movements incorporated in situations (struggle, pursuit, threat) acquire the nature of codes. It is still a problem to find out which codes of visual information are innate and which codes develop based on the former. In particular, there are many unclear issues regarding the origins of universals. For example, geometrical similarities of elements common for visual objects can be found in very different objects of visual information in various periods and cultures. This indicates that they take their origin rather in the systems of innate visual codes than in semiotic systems of culture, including in cultural codes of visual art. At the same time, proportions based on geometrical similarity are formed in culture, influenced either by patterns as their signs, or by structures of activity (constructive systems). In other words, universals of innate mechanisms of sensor information are concretized and turn into cultural mechanisms of perception - into cultural 
codes. This makes it very difficult to demarcate the aforementioned codes of group 5. Still, the differences between them are quite obvious.

The differentiation within the type paradigmatic-text codes (Figure 1, type 6) between paradigmatic codes (6.1), codes of structural expression (6.2), and codes of discourses (6.3) was carried out as one of the most important in some semiotic conceptions. The distinction between these codes can be regarded in visual information and visual art. Codes 6.1 are of a stable, repeated nature. Given the broadest interpretation of paradigmatics as relations of language-stable, repeated sign systems, these codes can include all codes 1 and 2, including those operating in the organization of a certain group of works of visual art, in particular, as canons or techniques. Codes 6.2 are formed and realized in communications due to the organization of certain groups of parts and relations within a certain object of visual information, a separate piece of visual art - in text structures of a picture (Schefer 1969:167). Organization of codes 6.2 plays a special role in the works that have a developed verbal basis, concepts that mean famous cultural situations and obtain advanced author's implementation in the works. Typical manifestations of this organization of codes (6) and their interrelations can be revealed in the course of analysis of art masterpieces. They can be illustrated through the example of some peculiarities of the structure of Giotto's fresco Kiss of Judas. ${ }^{2}$ The image is organized by a structure of visually important parts. Active elements raised clubs and torches - are directed towards the center. The axes of these parts are directed towards the center, surrounding the figure and nimbus of Christ. This technique stresses Christ's figure as the center of the image. Rhythmical structures of lines impart the tension of the depicted situation. This technique helps form codes of structural expression of the image. A circle of rays forms a metaphor - a sunshine-like phenomena. The likeness of Christ's figure and sunshine symbolizes the shining of truth and good in this context. At the same time the lines of this shining are broken and aggressive. Structures of signs unite several codes of structural expression. The directions of lines form an important independent structure of signs. Thus, relations of semantically important elements are formed; active meanings of tension and confrontation between good and evil are expressed. Another mural of the chapel that depicts Christ bearing his cross is also organized by general codes of structural expression. This image forms a single code. Active inclinations are repeated and stress the most dramatic moments.

Only one group of codes of structural expression that is important in the artwork is mentioned above. The multiplicity of such features and relations of organized elements and relations promote the diversity of codes of structural

2 Giotto di Bondone, Kiss of Judas (1304-1306, fresco, Scrovegni Chapel, Padua). 
expression and formation of various connotations - the entire semiotic systematicity of the text of an artwork.

The universalism of codes as mechanisms of transformation of structures is the basis of transformations: stable paradigmatic semiotic systems $\rightarrow$ single text semiotic systems. Structures of expression form the structure of a text. They can be fixed as a mechanism of a code and turn into a paradigmatic structure. Mechanisms of fixation of codes of structural expression and their synthesis in communication can be regarded as the basis of discourse formation. These interrelations correspond to discourse-forming models in the study of ethnocultural communities (Dijk 1982, 1987). In semiotic systems of visual art, interrelations between codes 6.1 and 6.2 in the unity with their bases - heterogeneities - are manifested in discourses used by individual authors, schools, trends, and styles.

The differentiation within codes of language systems (Figure 1, type 7) between pre-languages codes (7.1), language codes (7.2), and codes of language expressions (7.3) follows from the differentiation between the two sign systems: pre-language and language ones. According to Kristeva (1984), the former systems precede the latter. Unconscious inclinations, non-verbalized parts, and relations prevail in pre-language systems. These sign systems are interrelated with fundamental life situations and are generated in the models of these situations; they materialize the pre-language ones. Based on models, codes acquire more definite features in communicative structures of texts and are consolidated in their stable units and sign formations. Manifestations of these two different sign systems can be found in the works of visual art. Underlying, hidden sign systems that are typical for various schools and individual authors of visual art are of a more fundamental nature. There exist quite definite and easy-to-describe systems of language type. In particular, some well-known and partly examined "languages" of schools and individual authors are manifested in typical signs of images, in types of signs, etc. The manifestations of this differentiation in an artwork need to be explained.

A classical genre painting contains a layer of sign systems that is easily revealed by specialists aware of the peculiarities of characters and plot in culture, languages of poses, gestures, clothes accepted in various cultures, semiotic peculiarities of styles and trends that influenced the painting. A piece of art can be regarded as a set of peculiarities typical for a language used by a particular master: typical either for an artist or for a period of his/her creative work, etc. These areas of analysis include certain examined signs and sign systems, icons of objects denoted in images, their importance, structures of semantic systems, major symbols of culture and the spiritual world of an artist. At the same time, the very author's message of an artwork contains sign systems that cannot be easily described as systems of language type, as verbalized and easy to understand. Such 
phenomena as expressions of force, unsatisfied desires, visual-emotional generalizations of life, feeling of individual memory, deeply personal memories and images do not create obvious language structures. And at the same time, the given sign systems form an underlying basis of artworks and are accessible for study. This can be illustrated by the peculiarities of human generalization in visual art. Generalization of human types, character traits, and inner states became the core idea of great masters who tend to cognize human spiritual world. This cognitive principle became most apparent in the seventeenth-century art, in portraits by Velazquez and Hals. In particular, features of codes of identification of people's peculiarities, tempers, traits, and individualities are brought to perfection in portraits by Hals. Features of deep reflection, thoughts, hesitation, and suffering are supplemented by other identification features: the portrayed people's attitude to life and other people, to their social position and activity. These features reflect individual traits (Hals). They turn out to be broader and more fundamental than the codes of identification that are formed in these or those languages of culture. Codes of identification of people, their inner spiritual world can appear in visual art via the tendency to generalize them without revealing their individual peculiarities. In particular, this tendency is typical in the works by Surikov, one of the greatest Russian masters of realistic art of the nineteenth and twentieth centuries. The tendency implies a special view of people, life, and historic events. People in his paintings were generalized due to codes of identification according to classes of features (beautiful, kind, caring women, people who suffer and feel sorry for each other; or those who gloat when other people are humiliated). Due to these codes, people in multi-figured historical paintings were depicted as symbolic oppositions of participants in historic events. To do so, the artist used structures, features, and relations that promoted the implementation of the aforementioned identification codes (Somov 2013). People in Surikov's paintings are de-individualized and typified (Allenov 1997). Underlying codes of identification of life situations - simplicity, sincerity, malignity, aggression, suffering, and joy - were supplemented by the people and traits that were wellknown to the artist since childhood. They acquired the codes of known garments, household items, and various ethno-historical details.

The difference in penetration into an individual inner world in paintings by great masters is similar to the difference between individual and general. It illustrates the existence of pre-language non-verbal codes. These codes, though, are specified in sign systems of paintings due to the use of codes of verbal level, various codes of life and culture, and codes of structural expression of images.

Underlying pre-language systems (similarly to language systems) are formed on the basis of codes, structures, and heterogeneities; they are related with their basis - system of neurons - communicative channel. They must include a consid- 
erable number of innate and non-verbal codes and instinctive desires, according to Kristeva. This can be proved by the fact that many processes included in human visual perception evolutionarily continue biosemiotic non-verbalized mechanisms of coding sensor information used by mammals (Somjen 1972). As follows from the semiotic interpretation of neurology and genetics, they are determined by innate structure and codes of levels of neurons and other substrate foundations of structures and codes.

According to the theory developed by Kristeva, a new worldview and new models of the world appear. New models generate new codes. These processes also take place in visual art in new models of the world and new images, new ways to implement them in communication. According to Kristeva, new models generate new mechanisms for the realization of texts - their communicative structures. These new communicative structures of texts with new codes of structural expression are formed. Hence, it should be reasonable to differentiate between the following: codes and structures of new models of a worldview; codes of communicative structures of texts; codes of separate groups of elements of text. The given mechanisms of codes appear as new images, views of the world with their codes $\rightarrow$ new sign systems of the perceived elements of artworks $\rightarrow$ new groups of elements, features, structures, and their codes. These codes of levels of structural expression are manifested in the structure of a piece of art. In Giotto's fresco, a model of the world is implemented. Hostile forces are directed towards Christ but they are forceless. Directed towards Christ, they are a connotation of the threshold of torture. Radial composition also expresses the shining of the sun. Judas who is approaching Christ seems to break through the shining center and opens the way to beating his teacher and to the Calvary. But the solidity of the center - Christ's figure and head - forms a powerful shining center. The anxious rhythm of spears expresses the bellicosity of the crowd and the entire tension of the situation. Accidental nature and irregularity of the angles of slopes is a structure expressing the dynamics, anxiety, and bellicosity of the crowd's mood. It is related to codes of danger, brusque movements, and unexpectedness. Respectively, structures of spears and their abrupt directions opposed to the center of the painting can be viewed at the three aforementioned levels of codes of structural expression. At the first level, structures and features of spears form a generalized worldview. At the second level, structures and features form a communicative structure of the text, enable interpretation of the situation in the depicted event - opposition of Christ and Judas's deceitful love and devotion. At the third level, structures are essential for connection between basic signs, semes, representamens, and signals of the painting. They help express the tension of the depicted situation and visually connect color areas of the image plane. Codes of structural expression serve the basis of inner relations of various referents, denotations and 
connotations in the works of visual art. Due to these codes, various layers of connotations with their own codes are formed, which are known as "connotative codes" (Danesi 1999).

At the same time, codes that are typical for visual perception can be both denotative and connotative. They promote the denotation $\leftrightarrow$ connotation relations. A masterpiece of the art of portrait, Lady with an Ermine, by Leonardo da Vinci can be adduced as an example (Somov 2008). The snout and paws of the ermine symbolize the Duchy of Milan and its duke, express the central connotation in the painting. The predatory contours of paws and claws and the pointed snout of the animal become the hidden configuration of the picture, which promotes the formation of the metaphor of the animal and the lady. In general, the features of the predatory animal form important details of the painting and create the basis of codes of structural expression. General organizing codes and structures form denotations and connotations that seem to turn one into another.

One of the fundamental differentiations is the distinction within the group of codes of communication channels (Figure 1, type 8) between non-verbal (8.1), verbal and linguistic codes (8.2), and visual codes (8.3). In a number of semiotic conceptions, non-verbal codes are regarded as the continuation of verbal codes. Actually, many of humanity's sign systems were formed on the basis of verbal sign systems. This is partly true for visual codes as well. It is reasonable to view visual perception as the realization of "pre-verbal $\rightarrow$ verbal $\rightarrow$ non-verbal visual codes." The first group includes sensor codes, incorporated into regulation mechanisms "organism - environment" (the impact of warmth or cold; perception of abrupt movements of objects as danger; emotional impact of color that is partly determined by neurophysiological mechanisms). Non-verbal codes (Figure 1, subtype 8.1) include various relations that are fixed in behavior. Territorial defense is based on the friend or foe features identification. Rules of behavior are studied in proxemics (Hall 1980 [1973]). This can be applied to wide range of codes. Sensor codes are viewed as common for the production of sensor information of mammals (Guiraud 1975; Somjen 1972). These and other facts and conceptions point out that many non-verbal codes precede verbal codes and aren't based on them. Diversified information based on non-verbal codes is conveyed in visual information. Regardless of the signified, perception of rapid movement is a code of danger. Non-verbal codes are fundamental and syncretical codes of sensor information that are evolutionally differentiated into the tactile, auditory, visual, and others. The involvement of non-visual non-verbal codes is essential in visual art. Visual codes proper are secondary in relation to basic non-visual non-verbal codes. For instance, such are the visible movements of dancing people that are a rhythmical continuation of music, rhythmical movements of galloping horses and falling stones that are expressed in the rhythms of visually active elements of 
an image in paintings by Vrubel (Somov 2006). Non-visual non-verbal codes are the basis of visual codes here. The distinctions between the sources of energy, rhythms, movements, borders of objects, and properties of surfaces are perceived by non-visual communication channels. But these distinctions "move" into the areas of the visual channel as well. When considering codes it was specified on participation of non-verbal sensory codes in visual perception of visual art (Eco 1968). Art professionals who were close to the ideas of Russian formalism described the general natural syncreticity of perception that brings people closer to nature (Ivanov 2008). To sum up, one can say that non-verbal visual codes are partly secondary following non-visual non-verbal codes. At the same time, verbal codes form the basis of other codes - secondary ones, including visual codes (Figure 1, subtype 8.3). Non-verbal codes can be intensified in combinations with verbal codes. Various territorial names intensify the relations between territories and their visual signs with events. The relations of territories also exist in the orientation of animals beyond verbal communication. All the more so, they also exist beyond culture as semiotic systematicity over the verbal one. At the same time, verbal semiotic systems that include verbal codes are quite independent in languages and texts of visual art. They are manifested in subjects, concepts, and hypertexts of artworks. Verbal semiotic systems participate in visual perception being developed as concepts, giving systematicity to visual formations.

The differences between codes are related to the processes of regulation. Regulation is the processes that repeat and develop in cycles. This requires distinguishing codes according to regulation levels in the "organism-environment" system. This systems usually includes: "selection of signal (information) response - regulatory impact on situation.” Information is selected based on the choice of important responses to the questions that are put to the environment. Responses are expressed by emotions; regulatory impact is expressed by actions. In terms of semiotic theories, we would refer to the widespread differentiation between the codes of responses and actions. Hence, the following codes can be singled out as regulatory codes (Figure 1, type 9): codes of information selection (9.1), codes of response (9.2), and codes of actions (9.3). This distinction is reflected in psychological concepts. Codes 9.1 are materialized in feelings and simple sensations; codes 9.2 are materialized mainly in emotional reactions. Codes 9.3 are materialized in actions. These differentiations can be found in the classification of the levels of meanings in cognitive semiotics (Brandt 2006). Codes of group 9 are associated with choosing from various options and with the related principle of neuronal network operation (Hoffmeyer and Emmeche 1991; Ivanov 2008). Therefore they are realized in dual structures (Ivanov 2008). In particular, the duality of emotional states reflects the choice with the "organism-environment" regulation and is connected with impacts on the environment. The world of living 
systems - the response to differences; there are no responses in the world of physics and chemistry (Hoffmeyer and Emmeche 1991). This phenomena is illustrated by the answers “yes," “no,” and “I don't know.” Naturally, these differences are based on fundamental living selections - the questions put to communication. This point reflects the semiotic theory of the dialogical nature that was typical for communication and sign systems from the outset (Hoffmeyer and Emmeche 1991). The questions put to the human environment are manifested in various dualities that are later fixed in codes. Dualities of intentions are connected with other sign systems and appear in dual structures of various levels (activity and force, freedom and captivity, good and evil, truth and lie, etc.). Codes of regulations are realized in the organization of visual information and pieces of visual art. Codes of information selection (9.1) can be characterized as a continuation of intentional codes (3.1). Information that is vital for an individual is selected in visual perception intentionally. It continues the basic intentions: needs, motives, aspirations. Thus, intentional codes of danger are continued in the codes that reveal aggression or neutrality, activity, the force of living creatures or natural forces. Codes of response (9.2) are realized basically in emotions. In stable systems "stimulus-response," positive and negative emotions are linked with stimuli as codes (rhythm-emotion, color-emotion, contrast-emotion, etc.). These codes enable rendering moods in visual art. Signs of emotional states - complicated combinations of emotions - integrate separate codes and signs. Various codes (rhythmics, dynamics, color, configurations, etc.) are integrated. In particular, color range is a typical sphere of integration. Color combinations are an important source of creating mood in the general impact of an artwork. These moods are created by stable codes. A sequence of signals produces events of various probability. Emotional reactions are formed in these sequences in their quantitative properties in time (the amount of information; Moles 1966). These phenomena also extend to visual information. Rhythmical sequences of elements are interrelated with emotions based on visual perception. In particular, the use of rhythm in the traditional views of architectural composition is based on these phenomena. Codes of actions (9.3) are realized in various movements of people (movements of hands and legs, falling and rising, ascension, stopping, walking, gait, running, flying, aggressive gestures, caressing, rhythms of dance, staying alone, leaving, escape, pursuit, throwing). Structures that are typical for actions (bending, extension, rhythms of movements, directions, cycles, etc.) become established as codes. They are involved in creating visual information and visual art. Features of running, dancing, caressing, rising, flying, falling can form the basis of organization of artworks.

The distinction between codes of various levels of integration is manifested in the distinctions between abstract and concrete. This differentiation includes both 
concepts and semiotic verbal systematicities. It can be easily illustrated in the parts of an image. Relations of centers or axes of signified objects or their configurations on the surface are relevant to the abstract level; configuration relations - to the hints; relations of the same objects as concrete things, animals, and humans - to the concrete level.

Codes of integration levels (Figure 1, type 10) are differentiated based on "abstract- concrete" levels. The notion "level of integration" is in line with the theory of artificial languages elaborated by Garwin. The previous analysis of sign systems of visual information and visual art clearly reveals three levels of integration. Three basic levels of integration are singled out, namely: 1) the level of abstract universalia; 2) the level of bundles or realizations; 3) the level of specific objects. Naturally, codes operate at each of these levels. Codes of universals (10.1) function in the relations of various abstract elements (groups of elements connected by symmetry, rhythm, repeated numbers, common direction, etc.). The fundamental processes of visual perception are carried out on the basis of these structures. Such codes prevail in abstract art and are related to some of its concepts ("Suprema" by Malevich, "Prouns" by Lissitzky). These codes also extend to the entire visual information and all works of visual art. A complicated depiction of real objects always contains abstract universalia (geometric bodies, axes, centers, lines, dots, etc.). Structures of connections between these elements are naturally included in the codes of works. Abstract universals are most typical for symbolic codes (subtype 4.3). The results of semiotic analysis of some masterpieces of visual art indicate the complicacy of such codes and sign formations. For example, such are the symbolic constructions of the icon Trinity by A. Rublev. Its abstract symbolic structures are related to Byzantine theological doctrines (Somov 2007a). Therefore, symbolic abstract universalia prevail in this work. Triangles and thrice-repeated uses of basic elements symbolize the triunity of the world. The structures of rotation and reflection symmetry represent the relations between the heavenly world and earth. Triple repetition of the chalice symbolizes the triunity of the sacrifice. Circular movement means the harmony of the space and connects elements of an image. Repeated configurations of wings and their lines creates the effect of the presence of invisible angels. This works also contains other abstract universalia of the symbolic level. These universalia form the basis of coding the basic symbols of the image. Codes of bundles and realizations (10.2) are related to a more specific level. The importance of describing bundles (realizations) in image identification theory for better understanding of codes is in describing combinations of separate features (and codes) that are interconnected at the integrated level: as classes of features (Fayermark 1974). The mathematical model contains a kind of hyperplane in the "feature space." An algorithm of such a hyperplane can be viewed as a code that determines a class of 
features - a code that connects bundles (realizations). In this case, it is the mechanism of the connection of bundles integrated by codes that is the code connecting elements and relations. In more specific words, combinations of separate differential features are connected on the basis of feature classes. Classes of features form the so-called "alphabet of classes" - the basis of codes of this level (10.2).

The level of bundles and realizations produces information of a more specific nature, as compared to the information of the level of abstract universalia. But various abstract universalia can be "embedded" into specific visual information. These universalia, in turn, have a various degree of abstraction (highly abstract or semi-abstract). This is manifested in specific works of visual art. A landscape, still life, portrait or a picture with a plot can incorporate abstract - non-figurative and semi-abstract - semi-figurative sign formations. The correlation between them has their own peculiarities in the works of various schools, trends, and authors. Some masterpieces of visual art are "constructed" as interprenetrating sign systems of various integration levels (Figure 1, type 10). The Peasant Dance by Bruegel the Elder can be adduced as a vivid example of such systems. Peasants' figures are organized by means of various universals (Somov 2009), which makes it easy to perceive the dancing figures. At the same time, some features of groups of elements and relations form hidden connotations. For example, the contours of the boots worn by the running couple remind one of organic formations - the floating X and Y-X chromosomes - and symbolizes the masculine and the feminine. These connotations are related to the symbolic meaning of dance as a circuit of life. This example illustrates that groups of elements and relations of various integration levels (abstract geometrical figures, semi-figurative hints, concrete contours of people's arms, legs, heads, garment pieces) can be arranged as elements of a single text in an image. Groups of elements and relations of various integration levels are included in a text due to various codes that form denotations and connotations (Danesi 1999). Codes of bundles and realizations (10.2) are integrated between each other into denotations and connotations, as can be seen in fine arts. Thus, a drawing is an integration of group of codes (basically into denotative sign formations). Contours of people, animals, plants, movements of objects and their directions are formed on the basis of combinations of differential features of visual heterogeneity. In particular, these features include areas of straight and rounded contours, angles of rotation - both sharp and blunt angles, breaking points or smooth turns of contours, salient or concave areas, etc. Combinations of these and other features promote the integration of codes into identification of objects or their properties. Color is also a means of integration. Codes of color, similarly to codes of drawing, are integrated in elements and relations. Integration of color codes in bundles is carried out through the signifier 
and the signified relations. The signified of drawing and color form more specific realizations and acquire a more specific nature in the process of integration.

Various ways to form sign systems of visual information develop in accordance with integration of features and codes. The practice of drawing is often separated from the practice of painting. Drawing is related to the central area of visual information. Codes of this group produce visual information about vital situations and objects (movements, boundaries of objects, their shapes, etc.). Painting is based on the subtle distinction between tints and their semantics and is involved in visual information in a rather connotative way. Therefore various sign systems of a more specific level are formed on the basis of integration of codes of drawing and painting (10.3). Integration of codes and formation of a more specific level are related to the number and quantitative correlations of features of heterogeneity, with properties of physical carriers that form communicative channels. For example, glazing techniques used in oil painting help designate object properties (heaviness, dynamics, stability, hardness, softness, elasticity, fluidity, etc.). This also creates new opportunities of modelling shapes of objects by light-and-shade, designating sources of light, half-light, transparence of the environment, glitter, and transparence of object surfaces. Naturally, there appear extended opportunities for various connotations. In graphic arts, integration of groups of features (and, therefore, codes) also increases the number of signs. For instance, pen-and-ink drawing can be combined with black watercolor spots. These various groups of elements and relations form various sign systems in an image. This differentiation was subtly used by great masters. For example, in Picasso's graphic works, the linear pen-and-ink drawing basically designates objects, while vague spots of black watercolor create ghostly shadows, thus creating a specific layer of connotation.

Sign systems of color are formed at the level of bundles of differential features. For example, a combination of several color areas pertains to meanings of each of these colors. A common sign formation is created in their unity, with certain color prevailing in an image. Distribution and combination of colors also form signs. First of all, the prevailing colors and their quantitative relationship promote this or that emotional impact produced by an image. Color combinations become signs of images, mood, individuals, cultural traditions. These regularities appeared mostly in the art of the early Renaissance. Combinations of color areas were realized in relations of configurations of various objects (garment pieces, architectural constructions, plots of land, sky, etc.) in a plane. These color areas produced certain emotional impact and mood. The art of eighteenth century saw a freer use of the colors that bore emotive meanings (without meaning to designate people, clothes, architectural fragments). These colors lost their contours and were used in light-and-shade sign formations. This color systematicity was 
realized in Caravaggio's religious paintings in a new way. Combinations of reddish-orange, reddish-brown, brown colors, sepia, light ochre, warm grey tints in Caravaggio's large paintings with gospel motifs form a color range creating a peculiar impact. Combined with strange lighting, half-light, vagueness of the contours located in the shadow of objects, i.e., with the level of the signified, the meanings of indefiniteness and mysteriousness are formed. Color codes generate new codes. New codes of a more concrete level are formed due to integration of codes. Techniques of organization of works promote this formation in the field of visual art. Thus, new cultural codes are formed in some trends of the nineteenth-century art due to specific techniques of an image. For example, these are codes that enable one distinguish between roughness and elegance, coarseness and aristocratism. They are formed on the basis of more elementary codes. Features of angularity of shapes and contours of color areas produce a rougher depiction of people, which can be identified as strong, coarse, etc. On the contrary, opposite features and codes (complicacy and vagueness of contours, subtle details, etc.) help express delicacy, elegance, aristocratism. The integration of these codes with iconic codes of this level produces impressive, memorable images depicting scenes of everyday life. In particular, such paintings include scenes of coarse peasant life by both Bruegels, domestic scenes by van Ostade, rough figures of Parisians by Daumier, characters created by Russian itinerants of the nineteenth century: Perov, Makovsky, Myasoedov, and others. In later art, the codes of roughening the images of people changed under the influence of new models of the world and human (in expressionism, surrealism, and conceptual art). However, such basic codes are formed as integrations of identification mechanisms typical for visual perception.

The more bundles of differential features are integrated due to codes, the more specific the images become. The entire visible specificity is generated by codes. Given that, the functioning of the two main groups of codes: those functioning through the signified and the signifier and at the level of bundles should be taken into account. Codes of patterns (10.3) are mechanisms of relations between objects perceived directly (visible objects). They include sign formations that people perceive directly (unlike elements and relations of levels: 10.1 and 10.2). But what is directly perceived by people can be described in various ways. The objects that are perceived directly appear as visible things and their usual forms. These visible conventional forms are based on patterns - sign formations. Such formations are most clear as systems of behavioral reactions and actions in a certain type of life situations. These patterns of actions include systems of relations of the signified objects with life situations. Habitual relations and traditions are included. "Structure of mythological thinking," tropes, and other cognitive semiotic systems can serve examples of these patterns. Patterns are sign systems 
that are formed on the basis of integration of features and codes of abstract universalia (10.1), bundles and realizations (10.2). Images of people are associated with specific visible, easy-to-identify objects (as people and as silhouettes). At the same time, they correlate with each other in a conventional symbolic code. It is clear that their actual relations are integrated as several types of codes: identification (3.2), iconic (4.1), symbolic (4.3), codes of patterns (10.3), codes of images (11.2). This description can be applied to any elements and relations of visual information and visual art. They are manifested in sign formations as patterns in paradigmatics. Stable patterns in paradigmatics require no transformations. Therefore they are economical in text actualization and don't need any energy costs. Pattern is used as a simplified model of cognition, emotional reaction, and impact on situation. Patterns can be easily turned into clichés. These simplified patterns can be seen most clearly in standard architectural forms, ornaments, fashionable models of clothes, etc. Hence, these sign formations facilitate the identifiability and availability of texts. The use of these patterns does not require any changes in the mechanisms of mental activity. Patterns are typical images and forms of visual information and visual art, ways to simplify thinking, cognition, and communication. They are easily involved in mass communication processes and even create communication. This popularity of clichés was regarded in semiotics as very useful for the formation of culture (Lotman and Grishakova 2009). Based on this quality, pattern-like formations often become symbols of ethnoses, religions, states, and other vital symbols. Mythological and real characters become social ideals both as the signified and as patterns that function in communication and are easy to identify and perceive. Laconic patterns limit the amount of information in communication processes as codes that ensure functioning of these patterns are based on combinations of few features, which accounts for the peculiarities of simplification. In particular, simplifications of characters are typical for totalitarian art. Their faces, poses, gestures, clothes, and silhouettes turn into clichés. These images seem to have come off the assembly line.

Codes of the "abstract-concrete" levels include various types of relations: direct relations, interrelations, interdependence. Hence, various types of formations are created. They can be described as follows: structural formations, integrities, and forms. The difference between them are clearly expressed in images. Structural formations are manifestations of codes 10.1, a set of relations that are organized structurally. Integrities are manifestations of codes of realizations (10.2), organized sets of elements and relations, which reflects the interpretation of integrity in linguistics (Sapir 1930). Similar sets of elements and relations can be found in visual sign systems as well. For instance, the aforementioned Lady with an Ermine by Leonardo da Vinci contains various features that are meaningful in connotations (claws, configurations of the figures of the lady and 
the animal) and form integrities - groups of elements and relations. All similar complicated icons are formed by bundles of differential features and are manifested as interrelated integrities of groups of elements and relations due to codes 10.2. Based on indirect connections and interdependance, more definite and specific formations appear that are usually called external geometric shapes. Unlike structural formations and integrities, these formations are specific and have clear boundaries. For example, these are shapes of people's figures, objects of nature and household items that are perceived visually and indicated in images. The boundaries of these objects are vague in some works of art. For example, such are clouds and contours of trees. These fuzzy contours are also shapes. Various masters and various schools produced their own organizing forms (vague shapes of people and objects submerged in shadows in Titian's paintings; vibrating bundles of energy in compositions by Tintoretto; figures of bathers vanishing in the air by Boucher, de Chavannes, and Renoir). The signified of these contours are connected with verbal systems thus acquiring clearness and becoming units of the specific level (10.3). The lexical level is formed, i.e., those signs and denoted objects that we see and easily associate with words, verbal language. This level creates a specific world that is visible by people.

Some semiotic theories distinguish between codes of reality, codes of image, and codes of painting. A more general view of this classification can help single out codes of various realities: codes of information about life reality, codes of formation of sign systems that describe this reality, and codes of reality generated by human imagination on the basis of the former two types of codes. In brief, codes of different realities (Figure 1, group 11) can be divided into the following: codes of life reality (11.1), codes of images (11.2), and codes of virtual realities (11.3). Codes of life reality produce information about the reality on the basis of various features, structures, relations. In particular, such are the codes that specify the distance and remoteness between the objects and the spectator, movements and directions of object movements. These situations, objects, and properties are representamens of other situations, objects. and properties. Codes 11.1 also produce information about people's intentions, mental condition (codes of poses, gestures, mimicry). In general, codes of life reality embrace all spheres of life and are involved in the creation of visual information. Codes 11.1 become active in the formation of works of visual art. In particular, this is clearly seen in the use of codes related to summing up features of actual sizes of objects in space. Based on them, various techniques of geometrical perspective and its activation in visual art are formed. Features that promote the realization of these codes (parallel lines and planes, vertical elements, centers, etc.) are essential to create the impression of the depth of space, its structure and size. That is why architectural motifs were popular in the formation of perspective in the art of the Renaissance. Architecture 
became a semiotic system representing space in painting. Codes of production of information about space based on color features and differences were developed in visual art in interrelation with the development of oil painting techniques. The use of glazing promoted the use of various techniques of depicting air, the "aerial perspective." These techniques had been evident in the works of the last great Venetian school master - G. B. Tiepolo. Later, Dutch landscape painters Constable, Turner, Delacroix, painters of the Barbizon school, and impressionists used new techniques. Other typical phenomena of the development of life reality codes in visual art are related to personal identification. Depiction of people reveals their inner emotional state, feelings, intentions, moral values, etc. Codes connect the features of poses, gestures, mimicry, features of eyes with human inner emotional states, and usual character traits. These features and codes form the signs of intentions, emotions, and relations of depicted people (confrontation, argument, reconciliation, conviviality, etc.). In turn, these signs become representamens of ever more hidden aspects of human psyche, which realized in portraits to the fullest extent. A system of these designates in a multi-figured painting is related to other designates (life situation and its concepts, verbal texts, general mood of a work, etc.). The peculiarities of these codes are expressed in the individual nature of perception of people by masters of visual art. On the whole, codes of life reality form the basis of perceptive images, representations of various life situations. Codes of images (11.2) are created in heterogeneity of the communicative channel. This channel comes up as a visual heteregeneity in the formation of visual information and visual art. All relations of the elements of this heterogeneity promote the formation of the codes of this type. Codes of images emerge either based on codes of life reality or as independent codes, which accounts for various peculiarities of visual art. Basic techniques of an image can be based on codes of life reality. In this case, structures, features, relations of these codes predominate over structures of codes of images. A number of schools and masters of visual art also exist that use codes of "visual plane" as a basis, i.e., features, elements, and relations of this plane. Evidently, this reflects a popular expression "the conventionality of visual art." Given the interrelation between codes of life reality and codes of images, some codes can become subordinate to other codes: various combinations and combinations of their structures (codes 11.2 and 11.3). In particular, these are the interrelations of codes in the techniques used for creating perspective (rendering distance in landscape painting). Such techniques included contrasts between small divisions of distant hills, walls, towers, large human figures, and other features of division (vertical folds of clothes, active color contrasts) in plain, conventional paintings of early Renaissance. Such techniques exist in landscapes by Duccio di Buoninsegna, Serlio, and Pollaiolo. These techniques, used to create the effect of remoteness of objects, 
were supplemented by techniques of activation of small details of distant objects (tree crowns, architectural forms). With these techniques, structures, features and relations of codes of perception of real life space are involved in the codes of image - in its "conventionality." But codes of level 11.2 are also formed as independent codes - in heterogeneity of the visual plane. The differences between objects in terms of size can serve an example. These differences are conditioned by the hierarchic structure of their meaningfulness - the conventionality that breaks the connection with direct perception of real objects. This technique is typical for all "conventional” images. The relationship between characters is determined by conventional symbolic code of level 11.2. Codes of virtual realities (11.3) are formed as a specific field in addition to codes 11.1 and 11.2, including its own codes. For example, the use of iconic and indexical codes of recognition of object properties and structures of life situations is quite natural for the formation of codes. To illustrate this point, it would be reasonable to adduce a simple situation as an example: the transformation of a real world into a virtual world. Illusions of airborne dust, mist, and dampness were created in seascapes. Sign means of forming the effects of distance in landscape painting denoted a different reality: celestial heights, sky as a specific imaginary sign world. It is filled with diving beings - flying angels, saints, etc. Air was also represented through depicting a whole range of these flying creatures. And vice versa, the intensification of background effect, vagueness of background made the presence of living creatures in the sky hardly noticeable. This phenomenon was based on earlier interpretations of sky as a special world filled with divine beings (flying angels, saints, demons, etc.; The Temptation of St. Anthony 3 by Grünewald). The transformation of the codes of images into codes of virtual reality is similarly clear. The aforementioned techniques were used to create symmetry and mirror reflection helped organize visual plane. But at the same time, these techniques also served the basis of hidden connotations that formed a different reality. All techniques developed in modelled space in plane painting are similar. Structures of simplified geometrical-like elements that organize the visual plane, dabs, thick lines, active color configurations, contours of shadows, and other similar elements in images enable the creation of a new virtual world. This trend was already mentioned in semiotics while regarding iconic codes (Eco 1968).

Finally, codes that are regarded in semiotics as possible create the basis of developing imagination and forming virtual realities. The extent to which their visual peculiarities are correlated with semiotic systems of generation of virtual

3 Matthias Grünewald, The Temptation of St. Anthony (1510-1515, oil on panel, $265 \times 141$ [139] cm, Musée d’Unterlinden, Colmar, France). 
realities, including with the idea of "possible-worlds semantics," is a separate task. The suggested code typology describes some important differences and peculiarities of language-type systems and texts of visual information and visual art.

\section{References}

Aczel, Amir D. 2006. Nicolas Bourbaki: Theory of structures. In The artist and the mathematician: The story of Nicolas Bourbaki, the genius mathematician who never existed, 289-338. New York: Thunder's Mouth Press.

Allenov, Mikhail M. 1997. Vasiliy Surikov [in Russian]. Moscow: Slovo.

Arnheim, Rudolf. 1974. Art and visual perception: A psychology of the creative eye. Berkeley, CA: University of California Press.

Barbyshev, Eugenij N. \& Georgij Yu Somov. 1972. Struktura i informaciya kak osnovnoe zveno avtomatizacii arhitekturnogo proektirovaniya [Structure and information as basic stage of automatization of architectural design]. In Elgen P. Grigoryev (ed.), Teoriya proektirovaniya i problemy avtomatizacii proektnoy deyatel'nosti [Design Theory and Problems of Automation Design Activity], 96-113. Moscow: Institut avtomatizirovannih sistem v stroitel'stve [Collection of Articles of the Institute of Automatized Systems in Building], OTRD.

Barthes, Roland. 1965. Le degre zero de l'ecriture. Suivi de Elements de Semiologie. Paris: Gonthier.

Barthes, Roland. 1968. Elements of semiology. New York: Hill \& Wang.

Bense, Max. 1982 [1965]. Aesthetica. Baden-Baden: Agis.

Bongard, Mikhail M. 1967. Problema uznavaniya [The problem of recognition]. Moscow: Nauka.

Brandt, Per Aage. 2006. Form and meaning in art. In Mark Turner (ed.), The artful mind: Cognitive science and the riddle of human creativity, 171-188. New York: Oxford University Press.

Buyssens, Eric. 1967 [1943]. La communication et l'articulation linguistique. Brussels \& Paris: Presses Universitaires.

Cassirer, Ernst. 1965. The phenomenology of knowledge (The philosophy of symbolic forms 3), Ralph Manheim (trans.). New Haven, CT: Yale University Press.

Damisch, Hubert. 1978. Six notes in the margin of Meyer Schapiro's words and pictures. Social research 44(4). 15-35.

Danesi, Marcel. 1999. The interconnectedness principle and the semiotic analysis of discourse. Applied Semiotics/Semiotique Appliquee 6(7). 394-401.

Dijk, Teun A. van. 1982. Towards a model of ethnic prejudice in cognition and discourse. Amsterdam: University of Amsterdam.

Dijk, Teun A. van. 1987. Episodic models in discourse processing. In Rosalind Horowitz \& Shirley Samuels (eds.), Comprehending oral and written language, 161-196. New York: Academic Press.

Eco, Umberto. 1968. La struttura assente [The absent structure]. Milan: Bompiani.

Eco, Umberto. 1984. Semiotics and the philosophy of language. Bloomington: Indiana University Press. 
Fabbri, Paolo, et al. 1976. Rassegna critica sulla nozione di codice. In Intorno al "Codice" (Atti del III Convegno della Associazione Italiana di Studi Semiotici [AISS] Pavia 1975), 151-222. Florence: La Nuova Italia.

Fayermark, David S. 1974. Zadacha prishla s kartini [The problem came from a picture]. Moscow: Nauka.

Gombrich, Ernst H. 2000 [1960]. Art and illusion: A study in the psychology of pictorial representation. Princeton, NJ: Princeton University Press.

Greimas, Algerdas J. \& Joseph Courtes. 1979. Sémiotique, dictionnaire raisonné de la théorie du langage, vol. 1. Paris: Hachette.

Guiraud, Pierre. 1975. Semiology, George Gross (trans.). London: Routledge \& Kegan.

Hall, Stuart. 1980 [1973]. Encoding/decoding. In Centre for Contemporary Cultural Studies (ed.), Culture, media, language: Working papers in cultural studies, 1972-1979, 128-138. London: Hutchinson.

Hoffmeyer, Jesper \& Emmeche, Claus. 1991. Code-duality and the semiotics of nature. In Semiotic modeling, 117-166. Berlin \& New York: Mouton de Gruyter.

Ivanov, Vyacheslav V. 2008. Dualnye strukturi v antropologii: kurs lektsii, April-May 2005. [Double-natured structures in anthropology: Course of lectures, April-May 2005]. Moscow: Russian State University for the Humanities.

Jakobson, Roman. 1976. Six lecons sur le son et le sens. Paris: Editions de Minuit.

Kristeva, Julia. 1984. The revolution of poetic language. New York: Columbia University Press.

Lazarev, Viktor. 1997. The Russian icon: From its origins to the sixteenth century, Gerold I. Vzdornov (ed.). Collegeville, MN: Liturgical Press.

Lefebvre, V. A. 1973 [1967]. Konfliktuyushchie Struktury [Conflicting structures]. Moscow: Radio. Lotman, Jurij M. 1977. The structure of the artistic text [Michigan Slavic Contributors 7], Gail Lenhoff \& Ronald Vroon (trans.), Ladislav Matejka (ed.). Ann Arbor: University of Michigan.

Lotman, Yuri M. \& Marina Grishakova. 2009. Culture and explosion, Paul Cobley (ed.). Berlin \& New York: Mouton de Gruyter.

Lukács, György. 1969. Az esztetikum sajatossaga [Specifics of aesthetic], 2 vols. Budapest: Akademiai Kiado.

Moles, Abraham A. 1966. Teoriya informacii i esteticheskoe vospriyatie [The theory of information and the aesthetics perception]. Moscow: Mir.

Morris, Charles W. 1971 [1939]. Esthetics and the theory of signs. In Writings on the general theory of signs, 415-433. The Hague: Mouton.

Mouloud, Noël. 1965. La psychologie et les structures. Paris: Presses Universitaires de France. Mouloud, Noël. 1969. Langage et structures: Essais de logique et de séméiologie. Paris: Payot. Mounin, Georges. 1970. Introduction à la semiologie. Paris: Minuit.

Nöth, Winfried. 1998. Handbook of semiotics, Thomas A. Sebeok (ed.). Bloomington, IN: Indiana University Press.

Onasch, Konrad. 1962. Das Problem des Lichtes in der Ikonomalerei Andrej Rublevs. Zur 600Jahrfeier des grossen russischen Malers, vol. 28. Berlin: Berliner byzantinische Arbeiten.

Onasch, Konrad. 1988. Das Gedankenmodell des byzantisch-slawischen Kirchenbaus. In Karl Christian Felmy \& Wolfgang Heller (eds.), Tausend Jahre Christentum in Russland, 539-543. Göttingen: Vandenhoeck und Ruprecht.

Piaget, Jean. 1968. Le structuralisme. Paris: Presses Universitaires de France.

Prieto, Luis. 1964. Principes de Noologie. Fondements de la Theorie Fonctionnelle du Signifie. The Hague: Mouton.

Prieto, Luis J. 1975. Etudes de linguistique et de semiologie generales. Geneva: Droz. 
Rosenfeld, Azriel. 1969. Picture processing by computer. New York \& London: Academic Press.

Sapir, Edward. 1930. Totality. Language Monograph 6. 6-28.

Schapiro, Meyer. 1969. On some problems in the semiotics of visual art: Field and vehicle in image-signs. Semiotica 1. 223-242.

Schefer, Jean-Louis. 1969. Scènographie d'un tableau. Paris: Seuil.

Shekhter, Mark S. 1967. Psihologicheskie problemy uznavaniya [Psychological problems of recognition]. Moscow: Prosveshenie.

Somjen, George. 1972. Sensory coding in the mammalian nervous system. New York: Meredith.

Somov, Georgij Yu. 2005. Semiotic systems of works of visual art: Signs, connotations, signals. Semiotica 157(1/4). 1-34.

Somov, Georgij Yu. 2006. Connotations in semiotic systems of visual art (by the example of works by M. A. Vrubel). Semiotica 158(1/4), 147-212.

Somov, Georgij Yu. 2007a. Semiotic systemity of visual art works (case study of The Holy Trinity by A. Rublev). Semiotica 166(1/4). 1-79.

Somov, Georgij Yu. 2007b. Structures and semiotic systems. Semiotica 167(1/4). 1-35.

Somov, Georgij Yu. 2008. The role of structures in semiotic systems (analysis of some ideas of Leonardo da Vinci and the portrait Lady with an Ermine). Semiotica 172(1/4). 351-417.

Somov, Georgij Yu. 2009. Metonymy and its manifestation in visual art works: Case study of late paintings by Bruegel the Elder. Semiotica 174(1/4). 309-366.

Somov, Georgij Yu. 2012. Codes, heterogeneities, and structures: Visual information and visual art. Semiotica 192(1/4). 219-233.

Somov, Georgij Yu. 2013. The interrelation of metaphors and metonymies in sign systems of visual art: An example analysis of works by V. I. Surikov. Semiotica 193(1/4). 1-36.

Sonesson, Göran. 1997. Bridging nature and culture in cultural semiotics. Lecture given at the Sixth International Congress of the IASS, Guadalajara, July 13-19.

Stepanov, Yuri S. 1971. Semiotika. Moscow: Nauka.

Trubetzkoy, Nikolai S. 1969 [1939]. Principles of phonology, Christiane A. M. Baltaxe (trans.). Berkeley: University of California Press.

Uspensky, Boris A. 1976. The semiotics of the Russian icon (Semiotics of Art 3), Stephen Rudy (ed.). Lisse: Peter de Ridder Press.

Whitehead, Alfred North. 1927. Symbolism, its meaning and effect. New York: Macmillan.

Zemsz, Abraham. 1967. Les optiques cohèrentes (La peinture est-elle langage?). Revue d'Esthétique 20(1). 40-73.

\section{Bionote}

Georgij Yu. Somov (b. 1946) is a professor at the Urban Design Institute in Moscow <georgij.somov@gmail.com>, home page: gsomov.com. His research interests include the problems of de-sign, composition in architecture, semiotics of visual art, and theoretical semiotics. His publications include "Conviviality problem in the structure of semiotic objects" (2004); "Semiotic systemity of visual artworks: Case study of The Holy Trinity by Rublev" (2007); "Structures and semiotic systems" (2007); and "The role of structures in semiotic systems (analysis of some ideas of Leonardo da Vinci and the portrait Lady with an Ermine)" (2008). 